\title{
Theoretical Study on Electronic Structure and Optical Performance of Nickel and Nitrogen Codoped Rutile Titanium Dioxide
}

\author{
Fei Wang, ${ }^{1,2,3}$ Lei Feng, ${ }^{4}$ Dongmei Zhang, ${ }^{4}$ Qingguo Tang, ${ }^{1,3}$ \\ Jinsheng Liang, ${ }^{1,3}$ and Dan Feng ${ }^{1,3}$ \\ ${ }^{1}$ Institute of Power Source \& Ecomaterials Science, Hebei University of Technology, Tianjin 300130, China \\ ${ }^{2}$ Key Laboratory for Palygorskite Science and Applied Technology of Jiangsu Province, Huaiyin Institute of Technology, \\ Huaian 223003, China \\ ${ }^{3}$ Key Laboratory of Special Functional Materials for Ecological Environment and Information, Hebei University of Technology, \\ Ministry of Education, Tianjin 300130, China \\ ${ }^{4}$ Qianian College, Hebei United University, Hebei 064400, China
}

Correspondence should be addressed to Fei Wang; luckyphilip@sina.com

Received 11 April 2014; Revised 7 May 2014; Accepted 7 May 2014; Published 24 July 2014

Academic Editor: Jiaguo Yu

Copyright (C) 2014 Fei Wang et al. This is an open access article distributed under the Creative Commons Attribution License, which permits unrestricted use, distribution, and reproduction in any medium, provided the original work is properly cited.

\begin{abstract}
The nickel doped, nitrogen doped and nickel + nitrogen codoped rutile titanium dioxide have been investigated by ab initio calculations based on density functional theory. The electronic structure and optical performance of different ions doping models are researched through the obtained results, which reflects that the band gap of nickel and nitrogen codoped system declines apparently; the decrease of electron-hole pairs separation and charge carriers recombination rate becomes more desirable. Moreover, the optical absorption curves of nitrogen and nickel codoped rutile titanium dioxide demonstrate the higher photoresponse for visible-light than that of nickel or nitrogen single doped. The above results could provide theoretical basis for further developing of titanium dioxide photocatalyst and related experimental studies.
\end{abstract}

\section{Introduction}

Titanium dioxide has characteristics of strong catalytic activity, a long lifetime of photon-generated carrier, high chemical and thermal stability, and low cost; it has become one of the most extensively utilized ideal photocatalytic materials [1-3]. Generally, titanium dioxide has three basic crystalline phases containing brookite, anatase, and rutile titanium dioxide. Anatase titanium dioxide has been widely studied, and rutile titanium dioxide as the most stable one should be further studied and applied. Unfortunately, rutile titanium dioxide has intrinsic wide band gap value of $3.0 \mathrm{eV}$, and it can be activated only under the ultraviolet radiation from sunlight with only a small portion of the solar energy, resulting in its quite low solar energy usage [4-6]. Therefore, the high efficient usage of visible-light has become one of the most urgent purposes for the photocatalytic materials, and a large number of related theoretical and experimental studies have been performed to regulate the electronic structure and optical performance for titanium dioxide.

At present, many efforts have been made in enhancing the photocatalytic performance of titanium dioxide, including transition metal doping, nonmetal doping, noble metal loading, organic dye sensitizing, and semiconductor compounding [7-11]. Among the above methods, ion doping has been regarded as one of the most effective ways. The previous studies have mostly focused on the single ion doping for titanium dioxide [12-15], but relatively few recent experimental works show that various ions codoping into titanium dioxide can enhance the optical absorption scope and photocatalytic activity. However, the above experimental ions codoping work has disadvantages of variable experimental conditions and sample fabrication methods, resulting in the difficulty in studying the ion doping effect and modification 
mechanism [16-19]. Alternatively, the computer simulation could conquer the experimental work drawback and be applied to analyzing the ion doping effects and modification mechanism deeply.

In this paper, the density functional theory plane-wave ultrasoft pseudopotentials method within the first-principles framework has been applied to investigating the electronic structure and optical performance of different ions doping models, including nitrogen and/or nickel doping rutile titanium dioxide. The corresponding properties of pure rutile titanium dioxide are also calculated as a reference. Based on the above mentioned work, the effect of these ions on the electronic structure and optical performance of rutile titanium dioxide has been also illustrated.

\section{Models and Computation Details}

A $2 \times 2 \times 1$ supercell containing twenty-four atoms for rutile titanium dioxide has been developed in the calculations. As shown in Figure 1, in nickel and nitrogen doped titanium dioxide model, one titanium atom and one oxygen atom were substituted by nickel and nitrogen atom, respectively. As a result, one supercell consisted of seven titanium atoms, fifteen oxygen atoms, one nickel atom, and one nitrogen atom; the atomic concentration of impurity was about $4.17 \%$ (atomic fraction) in total. The other models were developed using the almost identical way.

$\mathrm{Ab}$ initio calculations based on the density functional theory were performed with the Cambridge Serial Total Energy Package code in Materials Studio 5.5 provided from Accelrys Software Inc. $[20,21]$. The ultrasoft pseudopotential was selected to depict the interaction between electrons and the ionic core. The electronic exchange-correlation energy was handled with Perdew-Burke-Ernzerhof (PBE) function in the generalized gradient approximation (GGA) framework. The energy cutoff for the plane-wave basis was $340 \mathrm{eV}$ and Brillouin zone integrations were finished using a MonkhorstPack grid of $3 \times 3 \times 8 \mathrm{k}$-points [22, 23]. To obtain stable atomic configuration and accurate results comparable with experimental data, pure and various doped models were geometrically optimized by means of the Broyden-Fletcher-GoldfarbShanno (BFGS) algorithm. The convergence threshold for self-consistent tolerance was set to $1 \times 10^{-5} \mathrm{eV}$ per atom and atomic relaxations were conducted until the residual forces were below $0.03 \mathrm{eV} / \AA$. The above parameters were suitable for getting well-converged total energy and geometry optimization results. After the geometry optimization, the band structure, density of states, and optical properties of the optimized supercells were calculated. For optical properties calculations, polycrystalline models and "scissors operators" were adapted.

\section{Results and Discussions}

After the geometry optimization, the lattice parameters of the pure rutile titanium dioxide supercell are obtained as follows: $a=9.32939 \AA, b=9.32939 \AA$, and $c=2.966985 \AA$. The above lattice parameters are in good agreement with

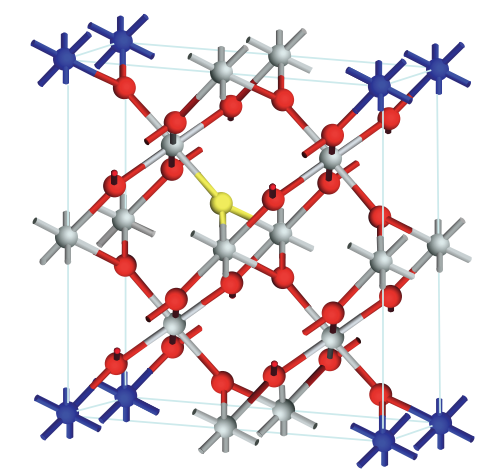

FIGURE 1: Supercell model for $\mathrm{Ni}+\mathrm{N}$-codoped rutile titanium dioxide in the present work (red ball represents the $\mathrm{O}$ atom; white ball represents the $\mathrm{Ti}$ atom; blue ball represents the $\mathrm{Ni}$ atom; yellow ball represents the $\mathrm{N}$ atom).

the experimental results $[24,25]$, which reflects that the chosen calculation methods are reasonable, and the authentic results could be obtained. Aiming at conveniently comparing the electronic structures of different ions doping models, the same k-points mesh is set to sample the first Brillouin zone for all models. The calculated band gap of pure rutile titanium dioxide is $1.87 \mathrm{eV}$ at $\mathrm{G}$ (gamma point) shown in Figure 2; however, the value is much lower than $3.0 \mathrm{eV}$ as the experimental value. The reason for the above phenomenon is that the discontinuity in the exchange-correlation potential is not taken into account within the framework of density functional theory, and the calculated band gap value is often lower than the experimental value $[26,27]$. The calculated band structures of $\mathrm{Ni}$-doped, $\mathrm{N}$-doped, and $\mathrm{Ni}+\mathrm{N}$-codoped rutile titanium dioxide are shown in Figure 2, respectively.

From Figure 2, we can see that the energy levels decline and are split due to the reduction of crystal symmetry degree and destruction of periodic potential field by means of doping. As a result, the valence band top and conduction band bottom of rutile titanium dioxide are both removed towards low energy. Meanwhile, the new energy levels between valence band and conduction band are introduced by electrons of impurity atoms, thus the band gap values of the doped system are all decreased. Specifically, the band gap decreases of $\mathrm{Ni}$-doped, $\mathrm{N}$-doped, and $\mathrm{Ni}+\mathrm{N}$-codoped rutile titanium dioxide doped system are $0.08,0.11$, and $0.44 \mathrm{eV}$, respectively. For Ni-doped rutile titanium dioxide, isolated impurity energy levels are mainly located in the middle of band gap. The above impurity energy levels overlap with valence band maximum or conduction band minimum of rutile titanium dioxide fully. For $\mathrm{N}$-doped rutile titanium dioxide, the impurity energy levels mainly located above the valence band are acceptor states. These states lead to the decrease of band gap and photoelectron transition energy. For $\mathrm{N}+\mathrm{Ni}$-codoped rutile titanium dioxide, the energy levels splitting becomes more apparent due to the further decrease of crystal symmetry degree, and more impurity energy levels are developed in the band gap of rutile titanium dioxide. Compared with the single $\mathrm{N}$ - or Ni-doped rutile titanium dioxide, the overlapping between impurity energy 


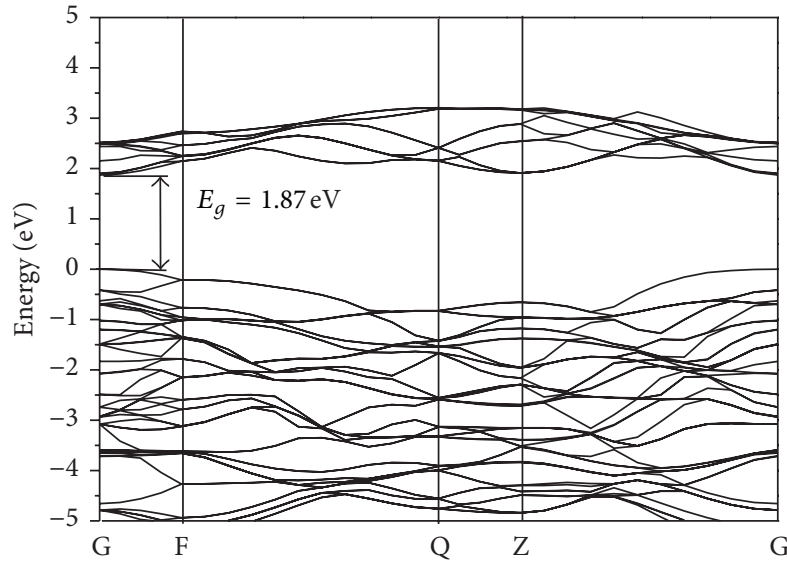

(a)

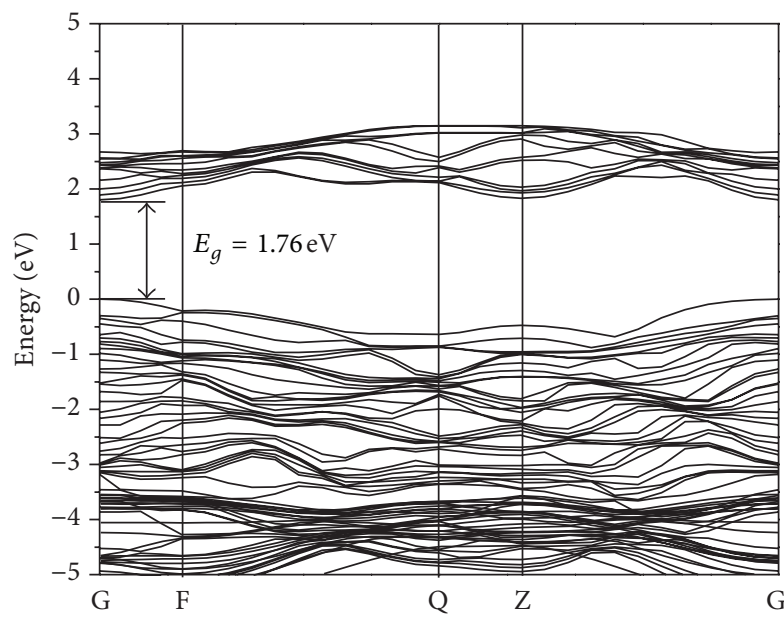

(c)

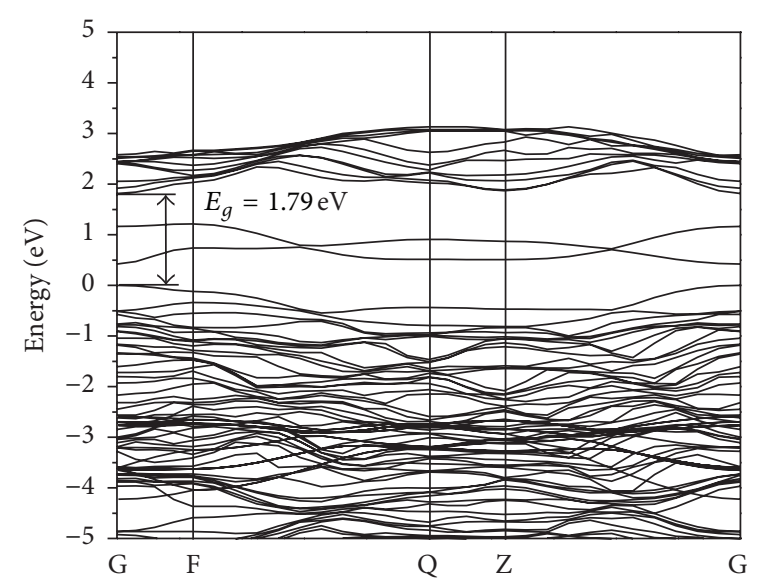

(b)

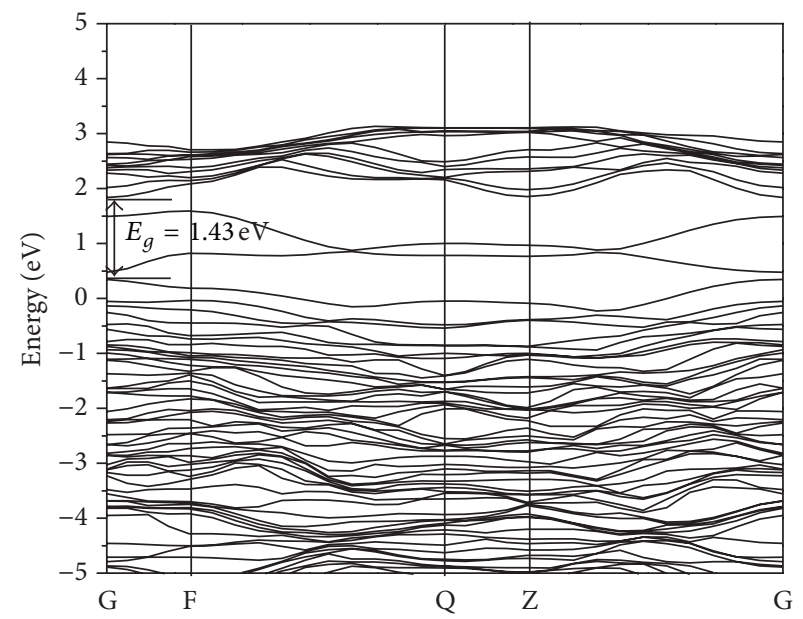

(d)

FIGURE 2: The calculated band structures of (a) pure rutile titanium dioxide, (b) Ni-doped rutile titanium dioxide, (c) N-doped rutile titanium dioxide, and (d) $\mathrm{Ni}+\mathrm{N}$-codoped rutile titanium dioxide.

levels and valence band maximum or conduction band minimum is more apparent. Nickel and nitrogen codoping can adjust the band structure of rutile titanium dioxide, and impurity energy levels are developed in the band gap of rutile titanium dioxide. As a result, the band gap of doped system is decreased effectively, and the separation of electronhole pairs becomes more desirable, which has significant influence on increasing the catalytic activity and visible-light absorption of rutile titanium dioxide [28, 29].

The calculated total density of states and partial density of states of doped models are shown in Figure 3, in order to analyze the origin of the band gap change and the variation of electronic structures caused by doping. Specifically, Figure 3(a) shows the total density of states of all the models; Figures 3(b)-3(e) represent the calculated partial density of states of different doped models, and the vertical dot line at $0 \mathrm{eV}$ is Fermi level.

From Figure 3, it can be seen that valence band and conduction band are mainly composed of $\mathrm{O}-2 \mathrm{p}$ states and Ti-3d states in all rutile titanium dioxide modles. For the pure rutile titanium dioxide, the conduction band is primarily provided by the $\mathrm{Ti}-3 \mathrm{~d}$ states, and the valence band is primarily given by the $\mathrm{O}-2 \mathrm{p}$ and partial Ti-3d states. The obtained electronic structures described in the present work are consistent with the results of other theoretical methods [30]. For Ni-doped rutile titanium dioxide, the impurity states are in the middle of band gap, which are composed of O-2p and N-3d states. These states service as a "ladder" through which the electrons in the valence band can be excited to them and then excited to the conduction band. For $\mathrm{N}$-doped rutile titanium dioxide, the valence band around Fermi level is composed by electrons in both the O-2p and N-2p orbit, and it is mainly composed by electrons in the O-2p orbit for pure rutile titanium dioxide. In addition, the valence band around Fermi level is wider than that of pure rutile titanium dioxide, and the valence band top has been shifted up. On the other hand, the conduction band width around Fermi level is approximate to that of pure rutile titanium dioxide, and the conduction band bottom has been shifted down. As a result, the band gap of the N-doped rutile titanium dioxide is decreased, which is consistent with the band structure calculated result. For nickel and nitrogen codoped titanium dioxide, the valence band is mainly 


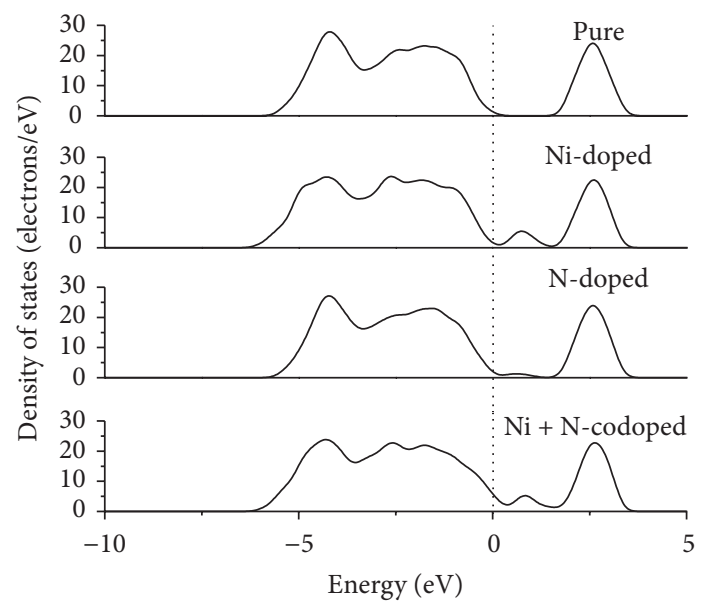

(a)

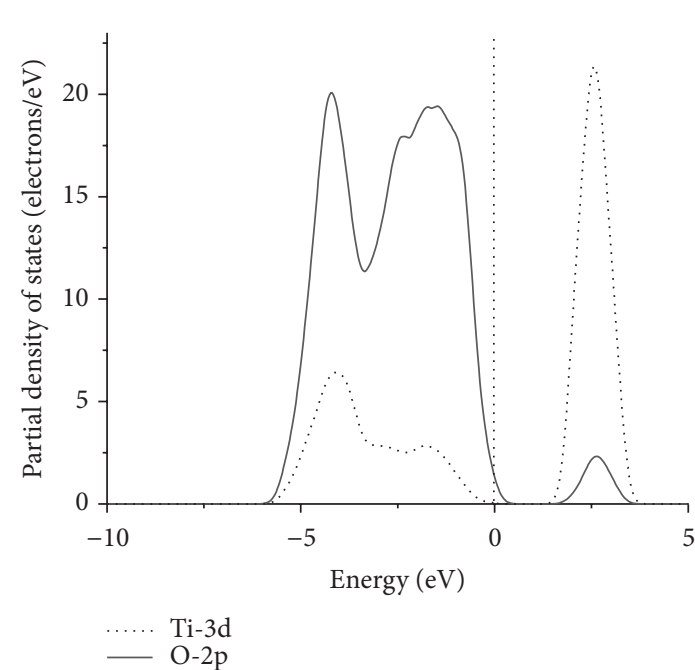

(b)

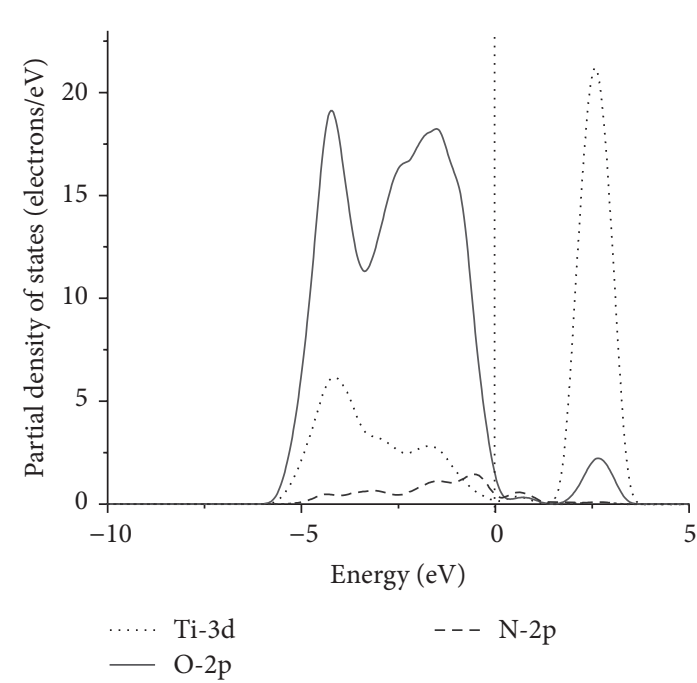

(d)

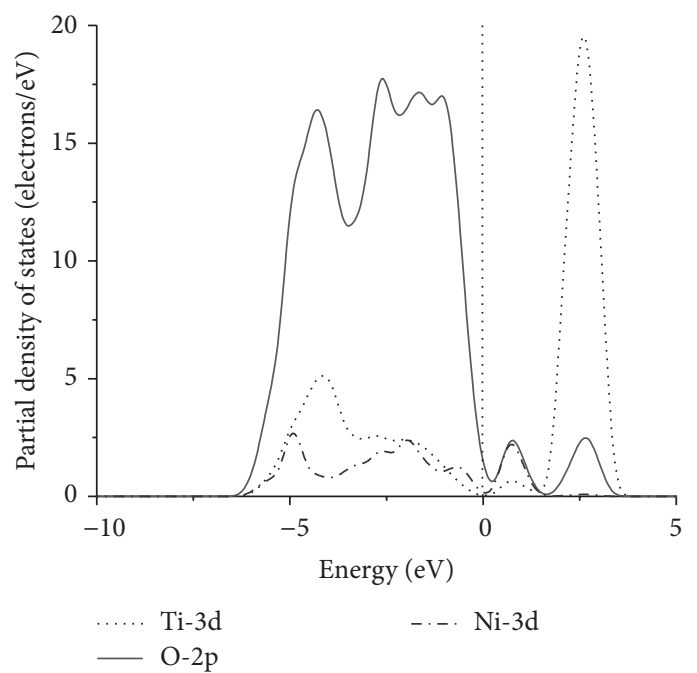

(c)

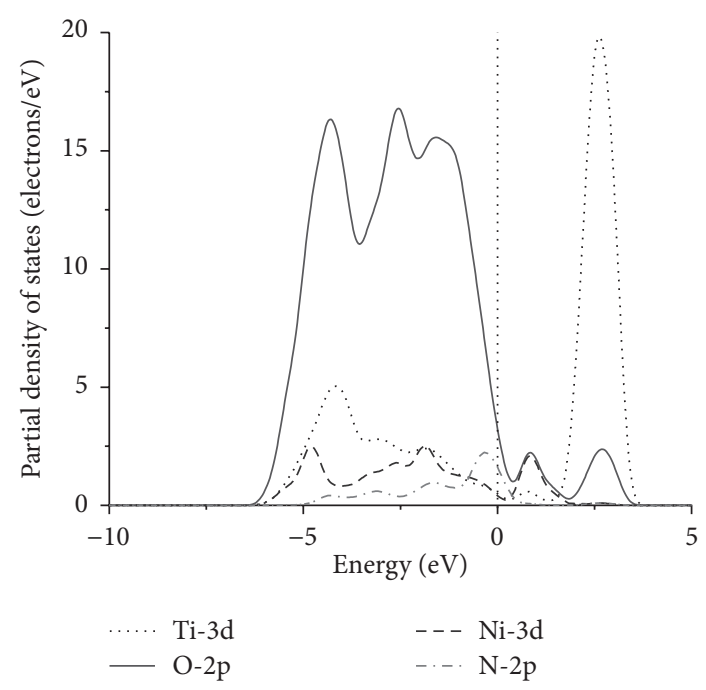

(e)

FIGURE 3: (a) Calculated and comparison total density of states; calculated partial density of states of (b) pure rutile titanium dioxide, (c) $\mathrm{Ni}$-doped rutile titanium dioxide, (d) N-doped rutile titanium dioxide, and (e) $\mathrm{Ni}+\mathrm{N}$-codoped rutile titanium dioxide. The dot line at zero point energy is Fermi level. 


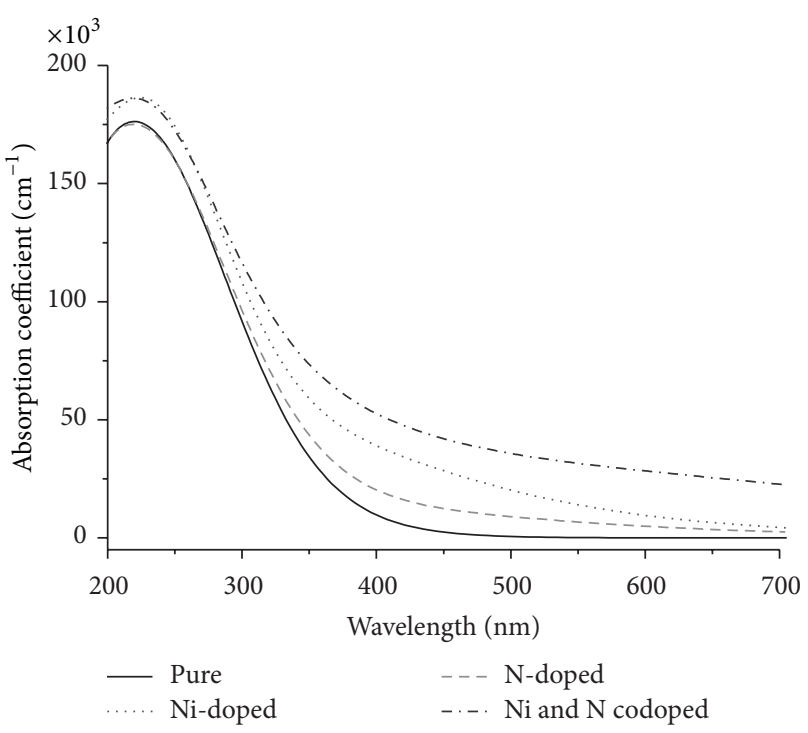

FIGURE 4: The calculated optical absorption curves for different kinds of rutile titanium dioxide models.

developed by O-2p states hybridization with $\mathrm{N}-2 \mathrm{p}$ states. The impurity energy levels developed by N-2p states overlapped with valence band maximum are situated on the valence band maximum, and the impurity energy levels developed by $\mathrm{Ni}-3 \mathrm{~d}$ states are situated on the top of valence band, resulting in the decrease of charge carriers recombination rate and photocatalytic activity improvement of titanium dioxide [3133]. Meanwhile, the electrons in the valence band can be excited to them and then excited to the conduction band subsequently by visible-light absorption, which is caused by the above impurity energy levels in the band gap. Therefore, these impurity energy levels have advantages on extending the sensitive light wavelength towards visible-light region.

The calculated optical absorption spectra of different doped titanium dioxide models between 200 and $700 \mathrm{~nm}$ are shown in Figure 4, in order to investigate the doping effect on the optical performance of rutile titanium dioxide. For all calculations, the scissors operator is chosen as $1.13 \mathrm{eV}$ based on the difference between the experimental and calculated band gap, which could make the obtained results consistent with experimental values.

From Figure 4, it can be seen that the fundamental absorption edges red-shift toward visible-light region after nitrogen or nickel doping. The above phenomenon is more apparent for nitrogen and nickel codoped rutile titanium dioxide than that of nitrogen or nickel single doped rutile titanium dioxide. Therefore, the optical absorption curves of nickel and nitrogen codoped rutile titanium dioxide indicate the highest photoresponse for visible-light, which is consistent with the conclusions obtained from the electronic structure analysis.

\section{Conclusion}

We have developed and calculated the supercells of pure rutile titanium dioxide, nitrogen, and/or nickel doping rutile titanium dioxide, using ab initio calculations with the planewave ultrasoft pseudopotentials method. On the basis of the above calculational results, the electronic structure and optical performance of the above various ions doping models have been also studied. The results indicate that the energy levels splitting becomes apparent for nitrogen and nickel codoped rutile titanium dioxide, and the overlapping between impurity energy levels and valence band maximum or conduction band minimum is more apparent than that of single ion doping. Nitrogen and nickel codoping is quite useful for the decrease of charge carriers recombination rate and energy gap, thus resulting in the great photocatalytic activity increase of rutile titanium dioxide. The optical absorption curves of nitrogen and nickel codoped rutile titanium dioxide indicate that the fundamental absorption edges red-shift toward visible-light region after nitrogen or nickel doping, especially for nitrogen and nickel codoped rutile titanium dioxide. Moreover, the highest photoresponse for visiblelight is consistent with the conclusions obtained from the electronic structure analysis. The above conclusions could give the theoretical advice for further developing of titanium dioxide photocatalyst and related experimental research.

\section{Conflict of Interests}

The authors declare that there is no conflict of interests regarding the publication of this paper.

\section{Acknowledgments}

This research was financially supported by the Natural Science Foundation of Hebei Province, China (Grant no. E2014202276), and the Application Foundation and Advanced Technology Research Program of Tianjin City, China (Grant no. 12JCQNJC02100).

\section{References}

[1] O. Ruzimuradov, S. Nurmanov, M. Hojamberdiev et al., "Fabrication of nitrogen-doped $\mathrm{TiO}_{2}$ monolith with well-defined macroporous and bicrystalline framework and its photocatalytic performance under visible light," Journal of the European Ceramic Society, vol. 34, no. 3, pp. 809-816, 2014.

[2] J. G. Yu, C. Trapalis, P. Y. Zhang, G. S. Li, and H. G. Yu, "Environmental photocatalysis 2013," International Journal of Photoenergy, vol. 2013, Article ID 786806, 3 pages, 2013.

[3] M. Erol, T. Dikici, M. Toparli, and E. Celik, "The effect of anodization parameters on the formation of nanoporous $\mathrm{TiO}_{2}$ layers and their photocatalytic activities," Journal of Alloys and Compounds, vol. 604, pp. 66-72, 2014.

[4] T. S. Bjørheim, A. Kuwabara, C. E. Mohn, and T. Norby, "Defects at the $\left(\begin{array}{lll}1 & 1 & 0\end{array}\right)$ surface of rutile $\mathrm{TiO}_{2}$ from ab initio calculations," International Journal of Hydrogen Energy, vol. 37, no. 9, pp. 81108117, 2012.

[5] T. K. Jia, F. Fu, J. W. Zhao et al., "Sonochemical synthesis, characterization, and photocatalytic activity of N-Doped $\mathrm{TiO}_{2}$ nanocrystals with mesoporous structure," International Journal of Photoenergy, vol. 2014, Article ID 516806, 7 pages, 2014. 
[6] C. C. Pei and W. W. Leung, "Enhanced photocatalytic activity of electrospun $\mathrm{TiO}_{2} / \mathrm{ZnO}$ nanofibers with optimal anatase/rutile ratio," Catalysis Communications, vol. 37, pp. 100-104, 2013.

[7] L. Ćurković, D. Ljubas, S. Šegota, and I. Bačić, "Photocatalytic degradation of Lissamine Green B dye by using nanostructured sol-gel $\mathrm{TiO}_{2}$ films," Journal of Alloys and Compounds, vol. 604, pp. 309-316, 2014.

[8] A. Kusior, J. Klich-Kafel, A. Trenczek-Zajac, K. Swierczek, M. Radecka, and K. Zakrzewska, " $\mathrm{TiO}_{2}-\mathrm{SnO}_{2}$ nanomaterials for gas sensing and photocatalysis," Journal of the European Ceramic Society, vol. 33, no. 12, pp. 2285-2290, 2013.

[9] K. Siwińska-Stefańska, F. Ciesielczyk, M. Nowacka, and T. Jesionowski, "Influence of selected alkoxysilanes on dispersive properties and surface chemistry of titanium dioxide and $\mathrm{TiO}_{2}-$ $\mathrm{SiO}_{2}$ composite material," Journal of Nanomaterials, vol. 2012, Article ID 316173, 19 pages, 2012.

[10] G. Kyung, M. Ha, and S. Ko, "Facile synthesis of solar light sensitive $\mathrm{Ag} / \mathrm{TiO}_{2}$ nanocatalyst wrapped with multiwalled carbon nanotubes," Nanoscience and Nanotechnology Letters, vol. 6, pp. 62-67, 2014.

[11] M. M. Rashad, E. M. Elsayed, M. S. Al-Kotb, and A. E. Shalan, "The structural, optical, magnetic and photocatalytic properties of transition metal ions doped $\mathrm{TiO}_{2}$ nanoparticles," Journal of Alloys and Compounds, vol. 581, pp. 71-78, 2013.

[12] K. Seonghyuk, "Photochemical synthesis, characterization and enhanced visible light induced photocatalysis of Ag modified $\mathrm{TiO}_{2}$ nanocatalyst," Journal of Nanoscience and Nanotechnology, vol. 14, no. 9, pp. 6923-6928, 2014.

[13] J. N. Xu, F. Wang, W. X. Liu, W. B. Cao, and J. Photoenergy, "Nanocrystalline $\mathrm{N}$-doped $\mathrm{TiO}_{2}$ powders: mild hydrothermal synthesis and photocatalytic degradation of phenol under visible light irradiation," International Journal of Photoenergy, vol. 2013, Article ID 616139, 7 pages, 2013.

[14] S. K. Zheng, G. H. Wu, and L. Liu, "First-principles calculations on $\mathrm{Hg}$-doped anatase $\mathrm{TiO}_{2}$ with and without O vacancy," Solid State Communications, vol. 165, pp. 15-18, 2013.

[15] M. Khan, J. Xu, W. Cao, and Z.-K. Liu, "Mo-doped $\mathrm{TiO}_{2}$ with enhanced visible light photocatalytic activity: a combined experimental and theoretical study," Journal of Nanoscience and Nanotechnology, vol. 14, no. 9, pp. 6865-6871, 2014.

[16] X. F. Wang, K. Wang, K. W. Feng et al., "Greatly enhanced photocatalytic activity of $\mathrm{TiO}_{2-x} \mathrm{~N}_{x}$ by a simple surface modification of Fe(III) cocatalyst," Journal of Molecular Catalysis A: Chemical, vol. 391, pp. 92-98, 2014.

[17] Y. R. Uhm, S. H. Woo, W. W. Kim, S. J. Kim, and C. K. Rhee, "The characterization of magnetic and photo-catalytic properties of nanocrystalline Ni-doped $\mathrm{TiO}_{2}$ powder synthesized by mechanical alloying," Journal of Magnetism and Magnetic Materials, vol. 304, no. 2, pp. 781-783, 2006.

[18] D. Wojcieszak, M. Mazur, M. Kurnatowska et al., "Influence of Nd-doping on photocatalytic properties of $\mathrm{TiO}_{2}$ nanoparticles and thin film coatings," International Journal of Photoenergy, vol. 2014, Article ID 463034, 10 pages, 2014.

[19] D. D. Sun, Y. Wu, and P. Gao, "Effects of $\mathrm{TiO}_{2}$ nanostructure and operating parameters on optimized water disinfection processes: a comparative study," Chemical Engineering Journal, vol. 249, pp. 160-166, 2014.

[20] Q. S. Shao and H. Zhao, "First-principles studies on magnetic stability of $\mathrm{SrC}$ and $\mathrm{BaC}$ in rocksalt, zincblende, and nickel arsenide phases under pressure," Journal of Superconductivity and Novel Magnetism, vol. 25, pp. 2063-2067, 2012.
[21] X. Guo, P. Q. La, X. F. Lu, Y. P. Wei, X. L. Nan, and L. He, “Theoretical research of electronic structures and optical properties of $\beta-\mathrm{Si}_{2}\left[\mathrm{Si}_{(1-x)} \mathrm{Yb}_{x}\right] \mathrm{N}_{4}(x=1 / 4,1 / 2$ and 3/4) based on the first-principles calculations," Nanoscience and Nanotechnology Letters, vol. 5, pp. 1237-1244, 2013.

[22] J. P. Perdew, K. Burke, and M. Ernzerhof, "Generalized gradient approximation made simple," Physical Review Letters, vol. 77, no. 18, pp. 3865-3868, 1996.

[23] J. G. Yu, Q. J. Xiang, and M. H. Zhou, "Preparation, characterization and visible-light-driven photocatalytic activity of Fedoped titania nanorods and first-principles study for electronic structures," Applied Catalysis B: Environmental, vol. 90, pp. 595602, 2009.

[24] J. M. R. Muir, D. Costa, and H. Idriss, "DFT computational study of the RGD peptide interaction with the rutile $\mathrm{TiO}_{2}$ (110) surface," Surface Science, vol. 624, pp. 8-14, 2014.

[25] X. Wang, F. H. Wang, J. X. Shang, and Y. S. Zhou, “Ab initio studies of $\mathrm{Nb}$ doping effect on the formation of oxygen vacancy in rutile $\mathrm{TiO}_{2}$," Journal of Physics and Chemistry of Solids, vol. 73, no. 1, pp. 84-93, 2012.

[26] J. Pascual, J. Camassel, and H. Mathieu, "Fine structure in the intrinsic absorption edge of $\mathrm{TiO}_{2}$," Physical Review $B$, vol. 18, no. 10, pp. 5606-5614, 1978.

[27] A. Dashora, N. Patel, D. C. Kothari, B. L. Ahuja, and A. Miotello, "Formation of an intermediate band in the energy gap of $\mathrm{TiO}_{2}$ by $\mathrm{Cu}-\mathrm{N}$-codoping: first principles study and experimental evidence," Solar Energy Materials and Solar Cells, vol. 125, pp. 120-126, 2014.

[28] P. Zhou, J. G. Yu, and Y. X. Wang, "The new understanding on photocatalytic mechanism of visible-light response N-S codoped anatase $\mathrm{TiO}_{2}$ by first-principles," Applied Catalysis B: Environmental, vol. 142-143, pp. 45-53, 2013.

[29] J. G. Yu, P. Zhou, and Q. Li, "New insight into the enhanced visible-light photocatalytic activities of B-, C- and B/C-doped anatase $\mathrm{TiO}_{2}$ by first-principles ", Physical Chemistry Chemical Physics, vol. 15, pp. 12040-12047, 2013.

[30] H. Yan, S. T. Kochuveedu, L. N. Quan, S. S. Lee, and D. H. Kim, "Enhanced photocatalytic activity of C, F-codoped $\mathrm{TiO}_{2}$ loaded with AgCl," Journal of Alloys and Compounds, vol. 560, pp. 2026, 2013.

[31] L. B. Xiong, J. L. Li, B. Yang, and Y. Yu, " $\mathrm{Ti}^{3+}$ in the surface of titanium dioxide: generation, properties and photocatalytic application," Journal of Nanomaterials, vol. 2012, Article ID 831524, 13 pages, 2012.

[32] Y. M. Lin, Z. Y. Jiang, C. Y. Zhu, X. Y. Hu, X. D. Zhang, and J. Fan, "Visible-light photocatalytic activity of Ni-doped $\mathrm{TiO}_{2}$ from $a b$ initio calculations," Materials Chemistry and Physics, vol. 133, pp. 746-750, 2012.

[33] F. Yuan, S. X. Lu, W. G. Xu, and H. F. Zhang, "First-principles study of structural, electronic, and optical properties of the rutile $\mathrm{TiO}_{2}(011)-2 \times 1$ surface," Surface Science, vol. 621, pp. 8893, 2014. 

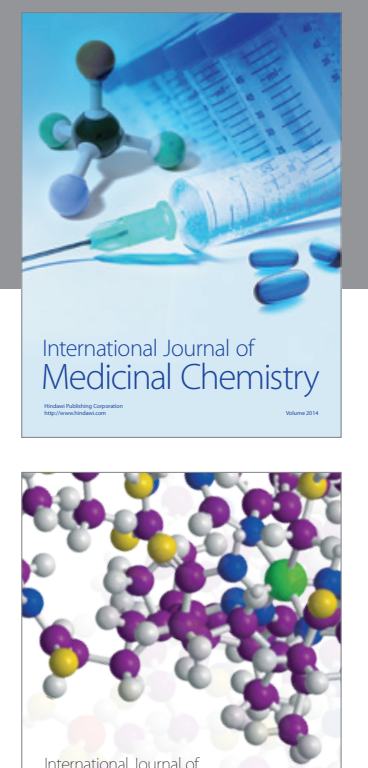

\section{Carbohydrate} Chemistry

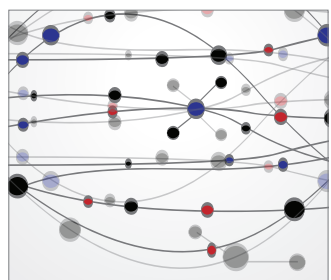

The Scientific World Journal
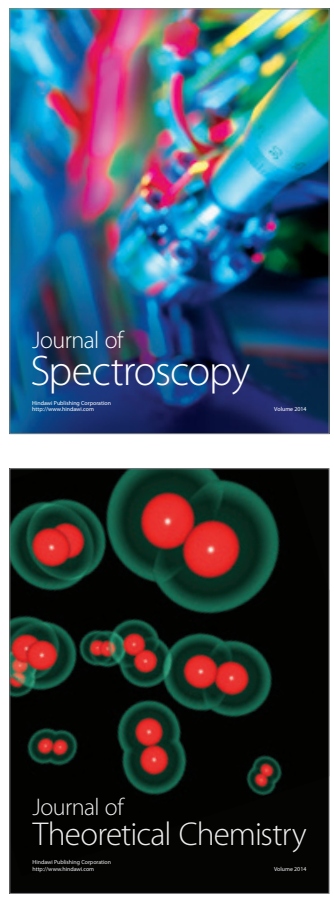
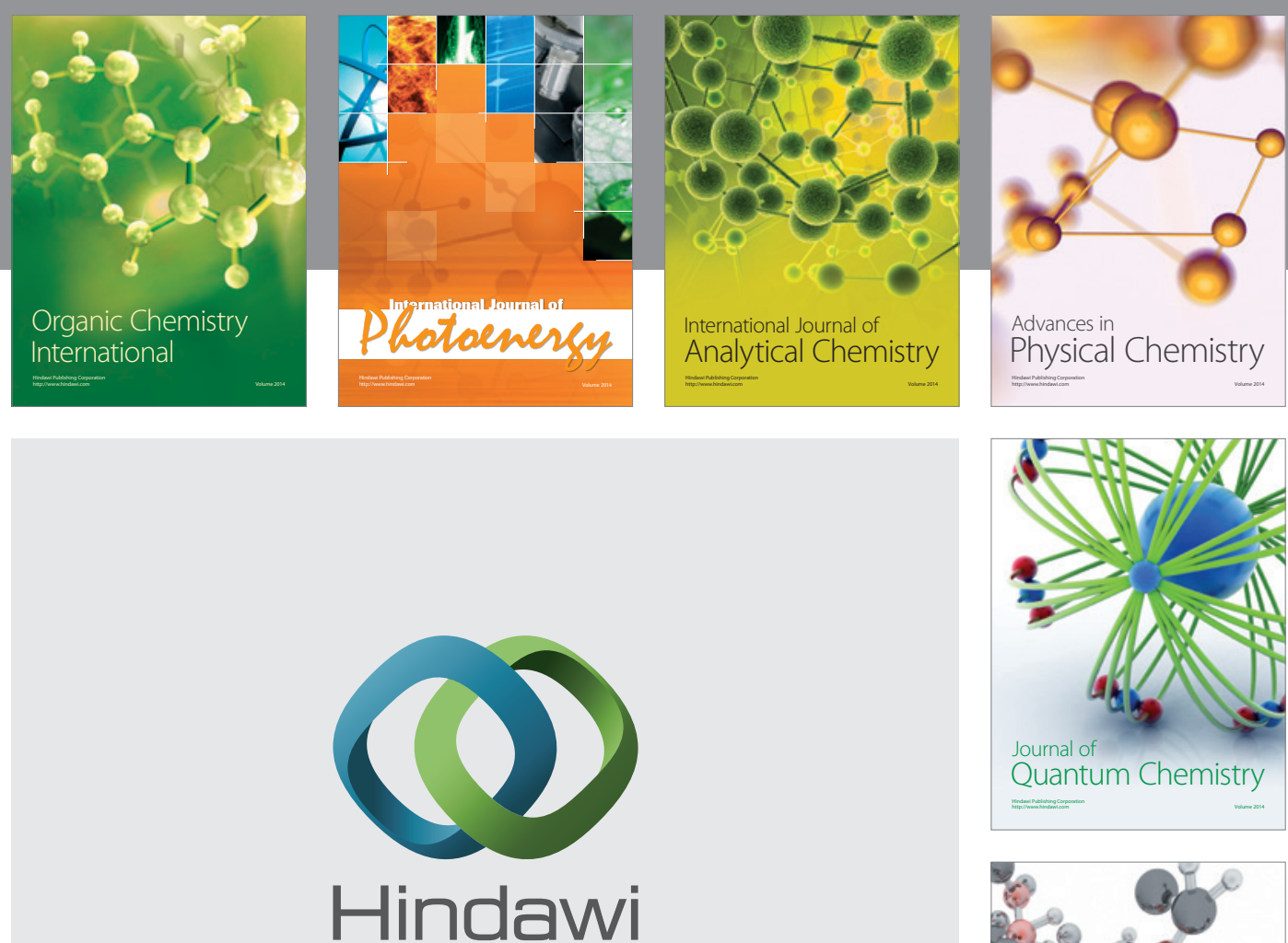

Submit your manuscripts at

http://www.hindawi.com

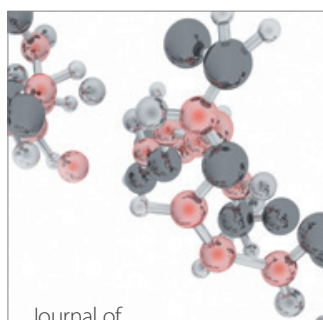

Analytical Methods

in Chemistry

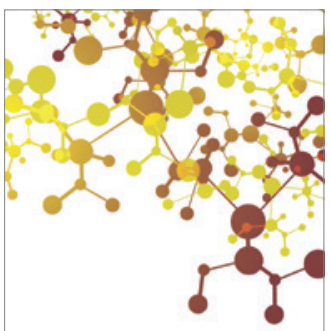

Journal of

Applied Chemistry

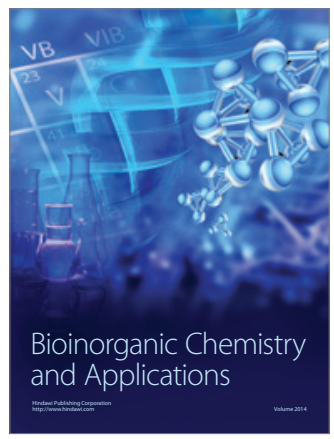

Inorganic Chemistry
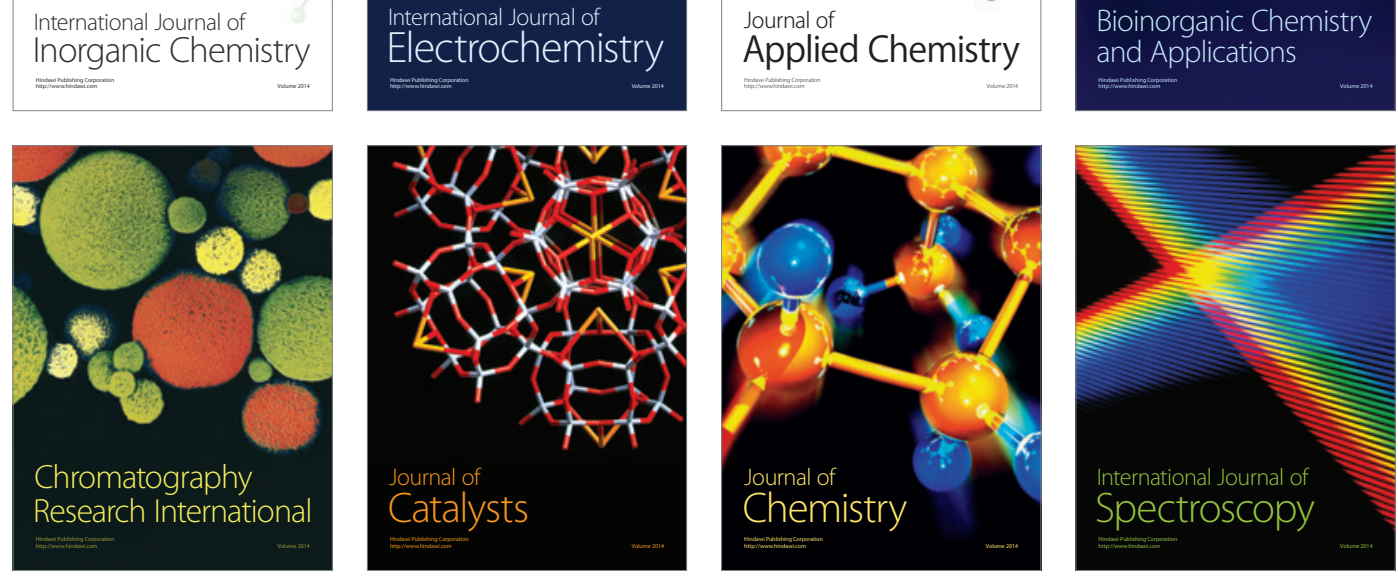